\title{
Déficit bilateral: origem, mecanismos e implicações para o treino de força.
}

\section{Bilateral deficit: origins, mechanisms, and implications for strength training}

\author{
Cíntia Ehlers Botton
}

Ronei Silveira Pinto ${ }^{1}$

Resumo -Vários estudos têm demonstrado uma menor capacidade de gerar força quando os exercícios de um programa de treinamento são executados bilateralmente em relação à soma da força executada pelos membros separadamente. Esse fenômeno, que tem sido bem descrito na literatura científica, é chamado de déficit bilateral. Os mecanismos causadores do déficit não são totalmente claros, mas parecem estar relacionados a mecanismos neurais, como um bloqueio dos hemisférios cerebrais ocorrido durante a contração bilateral que reduz a ativação de unidades motoras e a força produzida. Em relação a cada forma de execução (unilateral e bilateral) a longo prazo, as adaptações parecem ser específicas ao tipo de contração utilizada durante o treinamento. Portanto, em consideração à relevância da realização de exercícios executados unilateralmente e bilateralmente dentro de um programa de treino de força, assim como o efeito de cada forma de execução a longo prazo nas adaptações morfológicas e neurológicas do treinamento, esse artigo teve como objetivos caracterizar o fenômeno déficit bilateral, explorar seus principais mecanismos causadores, bem como discutir a influência de cada tipo de treinamento (unilateral e bilateral) nas adaptações do treinamento de força.

Palavras-chave: Eletromiografia; Força muscular; Treino de força.

Abstract - Several studies have demonstrated a lower capacity to generate force when the exercises of the training program are performed bilaterally than when compared to the sum of the strength produced by each limb separately. This phenomenon, one that has been well documented in the scientific literature, is called "bilateral deficit." The underlying mechanisms of the bilateral deficit are not fully clear, but they seem to be related to neural mechanisms, such as a blockage of the brain hemispheres, that may occur during bilateral contractions thus reducing the activation of motor units and therefore the strength produced. In relation to each execution form (unilateral and bilateral) in the long term, the adaptations appear to be specific to the type of contraction performed in the training. Therefore, due to the relevance of the exercises performed unilaterally and bilaterally in the strength training program, as well as the long-term effect of each execution form in the morphological and neurological adaptations to training, the aim of this article is to characterize the bilateral deficit phenomenon, to explore its main causative mechanisms, and to discuss the influence of each training type (unilateral and bilateral) in the adaptations of strength training.

1 Universidade Federal do Rio Grande do Sul. Laboratório de Pesquisa do Exercício. Escola de Educação Física. Porto Alegre, RS. Brasil.

Recebido em 06/03/12 Revisado em 24/06/12 Aprovado em 17/07/12

Key words: Electromyography; Muscle strength; Strength training. 


\section{INTRODUÇÃO}

Dentro da escolha dos exercícios para compor um programa de treinamento, é possível optar por exercícios que sejam executados unilateralmente (exercício realizado com um membro de cada vez) ou bilateralmente (exercício realizado com os membros homólogos simultaneamente). Vários estudos encontraram uma diferença nas cargas máximas entre exercícios executados de forma unilateral ou bilateral ${ }^{1-5}$, sendo que a soma das cargas máximas, de ambos os membros, na contração unilateral se mostra superior à carga realizada em contração bilateral. Esse fenômeno é denominado déficit bilateral (DB) e parece ocorrer em diferentes faixas etárias ${ }^{2,6,7}$ e grupos musculares ${ }^{2-4,8}$.

Alguns estudos têm demonstrado um declínio da atividade eletromiográfica em paralelo com o declínio da produção de força durante contrações bilaterais, mostrando que a redução de força, possivelmente, é causada pela menor ativação das unidades motoras quando os membros são exigidos simultaneamente ${ }^{4,5,9}$. Essa redução na atividade elétrica em contração bilateral é uma das hipóteses mais consistentes para justificar o $\mathrm{DB}$, uma vez que quando os dois hemisférios são ativados simultaneamente ocorre uma inibição inter-hemisférios limitando a máxima ativação das unidades motoras ${ }^{5}$.

Quanto às adaptações ao treinamento de força executado de forma unilateral ou bilateral, estas parecem ser específicas à condição realizada durante a rotina de treino, de forma que sujeitos que executam ações bilaterais parecem não estar sujeitos ao $\mathrm{DB}^{8}$. No entanto, são poucos os estudos que comparam as adaptações neurais e morfológicas decorrentes dos dois tipos de treinamento a longo prazo, observando a magnitude dos efeitos de cada tipo de treinamento.

Em consideração à relevância da realização de exercícios executados unilateralmente e bilateralmente dentro de um programa de treino de força, assim como o efeito de cada forma de execução a longo prazo nas adaptações morfológicas e neurológicas do treinamento, esse artigo teve como objetivo caracterizar o fenômeno DB, explorar seus principais mecanismos causadores, bem como discutir a influência de cada tipo de treinamento (unilateral e bilateral) nas adaptações do treinamento de força.

\section{PROCEDIMENTOS METODOLÓGICOS}

Realizou-se uma busca por artigos nas bases de dados Pubmed, Scopus e SportDiscus. A busca compreendeu todo período de existência das bases, até fevereiro de 2012, sendo que foi realizado um levantamento de artigos originais e revisões publicadas em revistas internacionais e nacionais. Para a busca, foram utilizadas as seguintes palavras-chave separadamente ou em combinação: déficit bilateral (bilateral limb deficit), treinamento de força (strength training) e exercícios unilaterais e bilaterais (unilateral and bilateral exercises). $\mathrm{O}$ número total de estudos encontrados em cada base 
de dados, de acordo com as palavras-chave, está na tabela 1. Como critérios de inclusão, foram considerados os estudos que se enquadravam nos seguintes aspectos: 1) estudos relacionados aos mecanismos causadores do DB; 2) estudos que avaliaram o DB em diferentes condições de teste e em diferentes populações; 3 ) estudos longitudinais que realizaram treinamento unilateral e/ou bilateral. Dentre o total de artigos, foram selecionados 49 para a leitura dos resumos, dos quais 30 se enquadravam nos critérios descritos acima e foram utilizados.

Tabela 1. Número total de estudos encontrados por palavra-chave em cada base de dados.

\begin{tabular}{lccc}
\hline & \multicolumn{3}{c}{ Bases de Dados } \\
\hline Palavras-chave & Scopus & PubMed & SportDiscus \\
Bilateral limb deficit & 386 & 252 & 50 \\
Unilateral and bilateral exercises & 435 & 438 & 24 \\
Bilateral limb deficit x Strength training & 11 & 10 & 8 \\
Bilateral limb deficit x Unilateral and & 19 & 16 & 2 \\
bilateral exercises & & &
\end{tabular}

\section{DÉFICIT BILATERAL}

Desde $1961^{10}$, estudos vêm demonstrando diferenças entre a produção de força máxima durante ações bilaterais quando comparadas às ações unilaterais, nas quais o somatório da produção de força dos membros contralaterais é superior à força produzida bilateralmente ${ }^{2-4,9,11}$. Segundo Owings e Grabiner ${ }^{2}$, essa diferença, chamada de déficit bilateral, é um fenômeno decorrente da redução do desempenho muscular durante movimentos bilaterais (membros sincronizados).

Estudos que investigam o DB têm como um dos interesses demonstrar diferenças na produção de força entre a execução bilateral e a soma da força de cada membro em execução unilateral. Essa diferença entre os dois modos de execução pode ser verificada pela equação de índice bilateral (IB) proposta por Howard e Enoka ${ }^{8}$,em 1991, e utilizada em outros estudos ${ }^{1,2,712}$ :

$\mathrm{IB}(\%)=\left[100 \mathrm{X}\left(\right.\right.$ forç̧a BL/ força $\mathrm{UL}_{\text {direita }}+$ força $\left.\left.\mathrm{UL}_{\text {egquerda }}\right)\right]-100$

$\mathrm{Na}$ qual são inseridos os valores da força produzida em condição BL (força $B L$ ) e a soma do valor da força de cada membro na condição $\mathrm{UL}$ ( força $U L_{\text {direita }}+$ força $U L_{\text {esquerda }}$ ). Valores negativos estão associados a maiores valores da soma unilateral e caracterizam $\mathrm{DB}$, sendo normalmente encontrados valores de $6 \%$ a $25 \%$ de DB na força ${ }^{4,13}$. Maiores valores em contração bilateral resultam em valores positivos e caracterizam o que tem sido denominado de facilitação bilateral.

Os menores valores de força em condição bilateral já foram observados em diferentes grupos musculares, como os flexores do cotovelo, os adutores do polegar, os flexores do joelho e os extensores do joelho ${ }^{1-4,7}$. Contudo, o DB ocorre apenas em musculaturas e membros homólogos. Músculos não 
homólogos, quando ativados simultaneamente, como flexores e extensores de cotovelo, ou músculos de membros não homólogos, como flexores de cotovelo e extensores do joelho, não demonstram diferenças na produção de força entre a condição unilateral e bilateral ${ }^{4,8}$.

Em relação à idade, sujeitos de faixas de idade distintas têm se mostrado suscetíveis ao DB. Esse fenômeno tem sido observado desde adolescentes e jovens adultos até sujeitos de meia idade e idosos, mas para uma mesma faixa etária, muitas vezes, mostram-se contraditórios. Owings e Grabiner ${ }^{2}$ hipotetizaram que devido à redução da ativação de fibras rápidas com o processo de envelhecimento, homens idosos teriam menor chance de possuir DB. Contudo, os resultados do estudo dos autores acima foram de encontro à hipótese principal, apontando um déficit de $6 \%$ a $12 \%$ para a extensão do joelho. Contrariamente, em estudo semelhante, Häkkinen et al. ${ }^{14}$ não encontraram diferenças entre os valores de força unilateral e bilateral, também na extensão de joelhos, de homens e mulheres de meia idade e idosos.

Ainda, poucos são os estudos realizados com adolescentes e é desconhecido o comportamento do DB em crianças. Kuruganti e Seaman ${ }^{7}$ encontram déficit em adolescentes, durante a extensão de joelhos isocinética $\left(45^{\circ} / \mathrm{s}\right)$ e os valores, comparados aos de idosos e adultos, não mostraram diferença significativa, contrariando o que seria esperado em resposta ao diferente recrutamento de fibras entre as faixas etárias.

No caso de sujeitos já familiarizados e que realizam treinamento de força, principalmente, de forma bilateral, essa diferença entre movimentos unilaterais e bilaterais não se torna tão evidente. Howard e Enoka ${ }^{8}$ realizaram estudo comparando sujeitos destreinados, ciclistas e levantadores de peso. O grupo composto por ciclistas não apresentou déficit, enquanto o grupo de sujeitos destreinados apresentou. Os levantadores de peso deslocaram cargas mais elevadas no teste bilateral do que a soma das cargas do teste realizado unilateralmente, caracterizando uma facilitação bilateral. Esses resultados reforçam a ideia de que exercícios bilaterais reduzem o déficit, pois ciclistas e levantadores de peso utilizam movimentos bilaterais em suas práticas e não apresentaram tal fenômeno. Já os sujeitos que não praticam treino de força são mais suscetíveis a presença do $\mathrm{DB}$, pois não sofrem influência do treinamento.

Outros fatores também colaboram com a divergência de resultados como o tipo, a intensidade e a velocidade da contração. Segundo Jakobi e Chilibeck $^{15}$, estudos que não encontraram o DB podem ser limitados pela variabilidade da população, pela diferença nas metodologias utilizadas, pela randomização da sequência de testes ou a inadequada reprodutibilidade do teste dinâmico.

As contrações isométricas podem melhor ilustrar a presença do $\mathrm{DB}^{15}$, pois minimizam fatores que podem influenciar negativamente o resultado do movimento, sobretudo, quando são avaliadas as condições neurais por eletromiografia (EMG). A qualidade e a estabilidade do sinal EMG em condições isométricas, decorrente principalmente do movimento reduzido 
dos eletrodos, durante os testes, possibilita a avaliação mais adequada das condições neuromusculares ${ }^{16}$. Entretanto, condições dinâmicas são mais utilizadas no treinamento de força e caracterizam melhor os movimentos realizados no dia-a-dia.

Embora em contrações isométricas haja um maior controle das variáveis acima referidas, existem variações entre as magnitudes de DB (relativo à força) reportadas na literatura, como por exemplo, para o movimento de extensão dos joelhos. Howard e Enoka ${ }^{8}$ encontraram déficit de aproximadamente 7\%, em destreinados, mas não o encontraram em levantadores de peso e ciclistas. Schantz et al. ${ }^{4}$ não encontraram DB e, ao contrário, registraram facilitação bilateral de $4 \%$ em sujeitos treinados e destreinados. Koh et al. ${ }^{13}$ encontraram, em sujeitos destreinados, DB de aproximadamente $17 \%$ a $24 \%$. Enquanto Häkkinen et al. ${ }^{14}$ não o encontraram em homens e mulheres de meia idade e idosos, em estudo de Owings e Grabiner ${ }^{2}$ idosos apresentaram DB entre 6\% e 12\%.

Com isso, é possível observar que mesmo que o fenômeno DB esteja bem demonstrado na literatura, existem divergências e lacunas em relação a algumas situações de testes, principalmente, em condições dinâmicas e de teste submáximo.

\section{PRINCIPAIS MECANISMOS CAUSADORES DO DÉFICIT BILATERAL}

Os estudos encontrados na literatura científica que investigam o DB são inconclusivos sobre os reais mecanismos causadores do fenômeno, uma vez que apontam diferentes justificativas e os resultados se mostram divergentes entre os estudos. As hipóteses principais para justificar o DB são relacionadas a mecanismos neurais, como: a limitação neural, a co-ativação antagonista e a reduzida ativação de fibras do tipo II. Nessa revisão, serão abordados os três mecanismos citados.

\section{Limitação neural}

Os mecanismos para a causa do DB ainda não são bem conhecidos. Uma inibição neural durante contrações máximas bilaterais é considerada uma das causas mais consistentes para explicar os menores valores de produção de força nesta condição em relação à condição unilateral ${ }^{1,2,4}$. No entanto, $\mathrm{O}$ caminho neural para essa inibição não é bem claro. A maioria dos estudos indica uma origem supra espinal ${ }^{1,4,5,8}$, mas ao mesmo tempo o DB pode estar relacionado a reflexos espinais.

Oda e Moritani ${ }^{5}$ encontraram, além da menor força em contração bilateral, uma depressão na atividade cortical durante este tipo de contração, visualizada por meio de eletroencefalograma de 11 sujeitos destros na flexão de punho, demonstrando que a origem da inibição durante as contrações bilaterais está possivelmente no córtex motor.

Alternativamente, tem sido sugerido que o DB pode estar relacionado aos reflexos espinais inibitórios ${ }^{4}$. Neste caso, estímulos sensoriais aferentes de um membro podem inibir os neurônios motores que controlam o membro 
contralateral no nível da coluna vertebral durante as contrações bilaterais ${ }^{18}$. Mesmo que os mecanismos reflexos não sejam os principais mecanismos causadores do DB, eles podem colaborar para que o mesmo ocorra.

A contração muscular que ocorre em um lado do corpo é controlada pelo hemisfério cerebral contralateral e os dois hemisférios cerebrais são conectados por fibras nervosas comissurais. Uma das possibilidades é que por meio da interação entre hemisférios, ocorra uma inibição mútua dos dois hemisférios cerebrais durante as contrações bilaterais, pois nesse tipo de movimento os dois hemisférios são requeridos simultaneamente. Desta forma, quando um dos hemisférios está em atividade ele diminui a ativação do hemisfério oposto, causando uma redução da estimulação de unidades motoras e resultando em menor produção de força ${ }^{19}$.

Com isso, a força e a atividade elétrica apresentam, geralmente, uma relação linear de queda durante a condição bilateral, nas quais os menores valores de força produzidos podem ser justificados pelos menores valores encontrados no sinal $\mathrm{EMG}^{1,4,5,9,13}$. Contudo, alguns estudos não demonstram essa relação de linearidade, justificando que o sistema nervoso central seria capaz de ativar maximamente as unidades motoras durante a contração bilateral ${ }^{6,8,12}$. Howard e Enoka ${ }^{8}$ encontraram valores de força 9,5\% maiores na condição unilateral e valores de atividade EMG 1,2\% maiores na condição bilateral. Os autores justificam que a quantificação do déficit é baseada em pequenas mudanças nos valores de força, que não seriam acompanhadas pela queda dos valores de atividade elétrica, pois o método eletromiográfico não é suficientemente sensível a pequenas alterações na força.

Como pode ser visto, grande parte dos estudos suporta a ideia de que ocorre uma inibição neural durante contrações bilaterais. Possivelmente, as diferenças metodológicas não permitam um consenso que esclareça qual a origem dessa inibição durante as contrações bilaterais. Além disso, a maior parte dos artigos não apresenta uma explicação clara e minuciosa dos processos que medeiam essa limitação.

\section{Co-ativação Antagonista}

A co-ativação é a simultânea ativação dos músculos agonistas e antagonistas do movimento durante a contração voluntária e está relacionado à estabilidade $\operatorname{articular}^{21}$. Diferentes estudos levantaram a hipótese de que o déficit poderia ser explicado pela maior ativação da musculatura antagonista do movimento em condição bilateral, diminuindo a ativação da musculatura agonista do movimento e, consequentemente, reduzindo a produção de força nessa condição ${ }^{11-13,20,22}$. No entanto, poucos foram os estudos que realmente realizaram essa medição. Alguns envolvendo a musculatura extensora do joelho $\mathrm{o}^{3,11-13}$ e apenas um envolvendo flexores plantares ${ }^{23}$, investigaram o nível de co-ativação e sua relação com o DB. Desses estudos, nenhum apresentou diferença significativa na co-ativação entre as duas condições, uni e bilateral.

Estudo de Jakobi e Cafarelli ${ }^{12}$ relatou um aumento da ativação dos isquiostibiais, durante a extensão de joelhos, linear ao incremento de 
carga das contrações $(25,50,70,100 \%)$, mas os valores desse aumento não mostraram diferença significativa da co-ativação antagonista nas condições uni e bilateral. Entretanto, os autores também não encontraram uma diferença na ativação da musculatura agonista do movimento entre as duas condições, não ocorrendo DB na situação em questão. Corroborando os resultados dos estudos acima referidos, Koh et al. ${ }^{13}$ avaliaram a atividade EMG dos isquiostibiais e encontraram maior ativação da musculatura antagonista em condição unilateral, mesmo quando a contração isométrica foi realizada em rampa ou o mais rápido possível. Apesar disso os valores não apresentaram diferença significativa.

Em estudo recente, Kuruganti et al. ${ }^{22}$ afirmaram que a co-ativação dos antagonistas não é mecanismo causador do $\mathrm{DB}$, visto que não houve diferença entre a atividade dos isquiostibiais durante extensão isométrica de joelhos. Mesmo assim, outros autores ${ }^{20}$ que não fizeram essa medição acreditam que possa ser a co-ativação uma das causas do DB. Desta forma, é interessante que mais estudos verifiquem a influência da co-ativação antagonista, uma vez que todos os estudos foram realizados com a mesma ação muscular (extensão de joelhos) e sempre sob condição isométrica. Ainda, os estudos não mostram informações claras de como os valores de co-ativação foram obtidos.

\section{Redução da Ativação de Unidades Motoras de Alto Limiar}

A reduzida ativação de unidades motoras de alto limiar vem sendo investigada na literatura como possível fator causador do $\mathrm{DB}^{9,24}$. Estudos especulam que durante as contrações bilaterais ocorre uma inibição no recrutamento de fibras do tipo II. Testes de fadiga ou comparação de diferentes velocidades de teste são utilizados para a especulação dessa hipótese, sendo que a divergência entre resultados pode ser resultante do reduzido $\mathrm{n}$ amostral ou mesmo da metodologia utilizada nos estudos.

Testes de fadiga sugerem a contribuição das fibras musculares durante movimentos isométricos máximos contínuos, condição em que são obervadas mudanças na média ou na mediana da frequência do sinal $\mathrm{EMG}^{16}$. Este comportamento de decréscimo na frequência do sinal está associado à velocidade de condução do potencial de ação na fibra muscular ${ }^{25,26}$ que é geralmente corelacionada com as fibras musculares do tipo $\mathrm{II}^{27}$. Caso haja realmente uma redução na atividade das fibras do tipo II, em condição bilateral, a força não deve ser tão afetada nessa condição, já que as fibras tipo I é que são principalmente recrutadas para resistir à fadiga muscular. Sendo assim, um menor decréscimo na queda da velocidade de condução do sinal elétrico da fibra muscular e, consequentemente, menor redução de mediana da frequência pode ser observado durante condições bilaterais, por estas possuírem uma limitação na ativação das fibras tipo II.

Koh et al.$^{13}$ realizaram contrações isométricas máximas de extensão de joelhos, nas condições ramp (força máxima atingida gradualmente) e step (força máxima atingida o mais rápido possível). O déficit foi encontrado 
em ambas as situações, mas se mostrou mais significativo na condição step $(24,6 \%)$ do que para a condição ramp (17\%), sugerindo que a redução na ativação de unidades motoras de alto limiar pode ser responsável pela ocorrência do DB. A hipótese dos autores era de que a condição step seria mais suscetível ao déficit, já que nessa condição as fibras rápidas contribuem de forma mais significativa para que ocorra produção de força máxima o mais rápido possível.

Em investigação recente, Kuruganti et al. ${ }^{24}$ sujeitaram o sinal EMG coletado durante teste de fadiga de 30 segundos a uma análise espectral e não encontraram diferenças na queda da média da frequência, sendo que esta iniciou e terminou semelhante para as contrações unilateral e bilateral. Os próprios autores assumem o reduzido $\mathrm{n}$ amostral utilizado como uma desvantagem, além do que é possível que a utilização da média da frequência não tenha sido o melhor método para análise dos sinais EMG, uma vez que está variável está mais sujeita a ruídos do que a mediana ${ }^{16}$.

Dado que o DB pode ser causado pela inabilidade de recrutar completamente unidades motoras de alto limiar durante um teste bilateral, o DB não deveria existir em intensidades submáximas de esforço, porque o uso de unidades motoras de alto limiar deve ser mínimo em baixas intensidades. Outra contradição é que se realmente esse é um dos mecanismos causadores do DB, sujeitos idosos deveriam apresentar menores valores de déficit em relação a sujeitos jovens, uma vez que com o envelhecimento ocorre atrofia das unidades motoras rápidas. Entretanto, há observações de que o déficit existe tanto em intensidades máximas como em submáximas, contrariando a explicação de que o déficit seria resultado do reduzido recrutamento de fibras tipo II. Ainda, o DB tem sido encontrado em sujeitos idosos na mesma magnitude que em jovens. Resultados de estudo de Hernandez et al. ${ }^{28}$ mostraram que o déficit não é diferente entre idosos e jovens adultos e ainda que o DB parece ser maior em condições submáximas (14-18\%) do que em condição máxima (11\%), quando realizado teste isométrico de flexão de cotovelo. Os autores sugerem que os sujeitos tiveram maior dificuldade de realizar as ações bilaterais em condição submáxima e que a explicação sobre a redução na ativação de unidades motoras de alto limiar não pode ser usada para justificar o DB.

Diferentemente do que se propõe sobre a redução na ativação de fibras do tipo II, Secher et al. ${ }^{29}$ sugeriram que a redução na ativação de unidades motoras lentas foi responsável pelo déficit encontrado no estudo. Os autores bloquearam parcialmente, através de drogas, a transmissão neuromuscular das unidades motoras lentas, observando redução no tamanho do DB para o exercício leg press de forma isométrica. Quando as unidades motoras rápidas foram bloqueadas, não houve diferença significativa no tamanho do DB.

A contraposição desses estudos mostra que existem divergências na literatura quanto à influência do tipo de unidade motora recrutada sobre o $\mathrm{DB}$ e que ainda são necessárias mais pesquisas que avaliem esse mecanismo. 


\section{TREINO DE FORÇA E 0 DÉFICIT BILATERAL}

Visto que o DB é causado por limitações neurais que reduzem o recrutamento de unidades motoras, o treinamento, que por sua vez tem como uma das adaptações neurais o aumento no recrutamento de unidades motoras, pode diminuir o DB quando executado bilateralmente ${ }^{30-32}$. Entretanto, são poucos os estudos encontrados na literatura que avaliam os diferentes efeitos da execução unilateral e bilateral, ao longo do tempo, na produção de força e na atividade elétrica.

Os estudos que investigam a influência do treinamento unilateral e bilateral encontram aumentos na força para ambos os tipos de treino, e os ganhos parecem ser específicos ao tipo de execução utilizada durante o treinamento. Em relação ao efeito dos dois tipos de treinamento no DB, os estudos mostram apenas efeitos relativos às respostas neurais do treinamento. Poucas são as evidências que demonstram os efeitos relativos às adaptações morfológicas como a hipertrofia muscular.

Em estudo de Kuruganti et al..$^{30}$, um treino isocinético progressivo de seis semanas com indivíduos jovens e adultos velhos, que possuíam DB para extensão de joelho, sugeriu que a dificuldade no recrutamento de unidades motoras durante a contração bilateral pode ser diminuída com o treinamento. $\mathrm{O}$ treino teve frequência de três sessões semanais, executado de forma bilateral a $45 \%$ s no dinamômetro isocinético para o exercício de extensão e flexão de joelhos, mostrando, no final do período de treinamento, que houve uma redução no déficit dos extensores de joelho, mas não dos flexores. Possivelmente, tal redução ocorreu porque os exercícios bilaterais diminuem o bloqueio do córtex cerebral e o treinamento influenciou no aumento do recrutamento de unidades motoras durante a contração bilateral. Já a redução para os flexores não foi tão significativa quanto para os extensores, o que pode estar relacionado às cargas insuficientemente aplicadas aos isquiostibiais. Além disso, os resultados não mostraram diferenças significativas entre o sexo e a idade dos sujeitos.

Estudo de Häkkinen et al. ${ }^{33}$ com homens e mulheres de meia idade e idosos durante 12 semanas, em que o exercício de interesse era a extensão de joelhos, mostrou nos pós-testes, que os ganhos de força e de atividade elétrica muscular foram específicos. O grupo de treino bilateral aumentou de forma mais significativa a força e o sinal EMG nesta condição e o grupo que treinou unilateralmente teve aumentos mais significativos na condição unilateral. As adaptações específicas para cada tipo de treino são justificadas por bases neurais, considerando o aumento na ativação dos músculos agonistas do movimento. Além disso, nenhum dos sujeitos apresentava DB no início do treinamento, mas provavelmente pelas adaptações do treino, a razão força bilateral/unilateral aumentou $7 \%$ para os sujeitos que treinaram de forma bilateral e reduziu $2 \%$ para aqueles que treinaram de forma unilateral.

Contrapondo a ideia das adaptações serem específicas ao tipo de treino realizado, estudo de McCurdy et al. ${ }^{34}$, com homens e mulheres jovens, após oito semanas de treino unilateral e bilateral do exercício agachamento, mostrou aumentos de força em ambas as condições para os dois grupos. 
Como pode ser visto, apesar de existirem evidências de que o treinamento de força pode interferir no DB, seja aumentando-o (treino unilateral) ou diminuindo-o (treino bilateral), por meio da maior ou menor ativação das unidades motoras, dos poucos estudos que são encontrados alguns não avaliaram a atividade elétrica, apenas sugerindo que, possivelmente, as adaptações a cada tipo de treinamento tenham uma influência neural sobre o DB. Ainda, os estudos realizados não deixam claro se os sujeitos possuíam $\mathrm{DB}$ e se a amostra era homogênea em relação à magnitude do $\mathrm{DB}$, característica que pode interferir nos resultados do estudo.

Outro ponto a considerar é que a maioria dos estudos foram realizados com a população idosa, adultos velhos ou mulheres pós menopáusicas. Como já foi mencionado, em virtude de efeitos deletérios no recrutamento de fibras com o avanço da idade, é possível que os resultados possam não demonstrar com clareza a influencia do treino de força no fenômeno DB, já que o fator idade influencia os resultados. Além disso, a aderência de jovens aos programas de treinamento de força é cada vez maior e os efeitos do treinamento têm maior magnitude nessa população, como os aumentos absolutos da espessura muscular ${ }^{35}$.

Mesmo que os estudos realizados com treinamento indiquem que as adaptações acontecem por vias neurais, ainda faltam investigações que considerem outras adaptações neurais que não só o aumento da ativação da musculatura agonista. Considerando que uma adaptação neural ao treinamento de força é a redução da co-ativação antagonista e que durante a condição bilateral pode existir uma co-ativação mais acentuada, uma das explicações para redução do DB com o treino bilateral poderia ser a redução da atividade dos antagonistas do movimento. No entanto, nenhum dos estudos encontrados na literatura realizaram essa observação, de forma que ainda não se tem informações sobre o efeito de cada treinamento na co-ativação bem como sua comparação.

Quanto às adaptações morfológicas, uma das adaptações fundamentais do treino de força é o aumento da massa muscular, que caracteriza a hipertrofia muscular. Em teoria, esse aumento pode ocorrer como resultado de um aumento na fibra muscular, um aumento no número de fibras ou pelo aumento da quantidade de tecido conectivo no músculo ${ }^{36}$.

A maioria dos artigos referidos nessa revisão, relativo ao treinamento de força unilateral e bilateral, não comparou as adaptações morfológicas decorrentes dessas duas formas de treino. Há, então, uma lacuna na literatura quanto ao aumento da espessura muscular pós-treinamento de força uni e bilateral, assim como sua comparação.

Kuruganti et al. ${ }^{30}$ utilizaram a medição da dobra cutânea da coxa direita pré e pós treino bilateral, para identificar se houve aumento da espessura muscular. Foi constatado que não houve aumento na espessura da dobra cutânea. No entanto, o tipo de método (antropométrico) apesar de ter sido executado com precisão, é apenas uma medida indireta. Dessa forma, sem dados que relatassem contribuição das adaptações morfológicas, eles sugeriram que os aumentos de força máxima no estudo foram causados por adaptações neurais. 
Häkkinen et al. ${ }^{33}$ utilizaram a técnica de ultrassonografia para analisar a área de secção transversa do vasto lateral, de homens e mulheres de diferentes idades que treinaram de forma uni ou bilateral. Os aumentos na área de secção transversa, de ambas as pernas para quem treinou de forma bilateral foram significativos para quase todos os grupos, não sendo apenas para homens de 50 anos, os quais incrementaram apenas a área da perna esquerda. Quanto ao treino unilateral, os aumentos na área de secção transversa foram significativos para todos os grupos. Os resultados do estudo não mostraram diferença significativa entre o aumento dos grupos uni e bilateral, visto que houve um aumento relativo de $14 \%$ na média da área de secção transversa, de ambas as pernas, nos grupos que treinaram bilateral comparado ao aumento de $11 \%$ na média do grupo unilateral. Além disso, não houve correlação significativa entre os aumentos da massa muscular e as mudanças nos valores de $1 \mathrm{RM}$ para cada grupo.

Janzen et al. ${ }^{31}$ utilizaram Raio X para verificar as mudanças na massa muscular de mulheres pós menopáusicas que treinaram de forma ui ou bilateral. Em relação ao grupo controle, o grupo unilateral aumentou de forma significativa a massa muscular. Entretanto, não foram encontradas diferenças entre o grupo unilateral e bilateral para os extensores do joelho. Os autores hipotetizaram que o treino unilateral aumentaria de forma mais significativa a massa muscular por permitir o levantamento de cargas mais altas. Em parte, a hipótese foi considerada, pois apesar dos ganhos de massa não terem sido diferentes entre grupos, para o exercício extensão de joelhos, as cargas levantadas realmente foram maiores no grupo unilateral.

Assim como o estudo de Häkkinen et al. ${ }^{33}$, o estudo de Janzen et al. ${ }^{31}$ foi realizado com idosos e possivelmente os efeitos deletérios do envelhecimento tenham dificultado a observação de diferenças no incremento de massa muscular. No caso do estudo de Janzen et al. ${ }^{31}$, embora tenha ocorrido aumentos da massa muscular para o grupo unilateral, esses foram pequenos (4\%) em relação aos aumentos na força (25\%).

Por isso, seria interessante a realização de estudos que investiguem a população jovem e possam comparar qual dos dois treinamentos gera maiores incrementos, sem interferência de fatores como a idade. Assim, a execução uni ou bilateral dentro de um treinamento poderá ser utilizado, quem sabe, como forma de implementar as adaptações do treinamento de força de forma específica.

\section{CONSIDERAÇÕES FINAIS}

O déficit bilateral é um fenômeno que merece atenção, pois caracteriza uma limitação neural durante contrações bilaterais que influencia a capacidade de gerar a força máxima, dificultando ou não o desempenho na atividade que está sendo executada. A ocorrência desse fenômeno é vista em diferentes faixas etárias, grupos musculares e em situações isométricas, isotônicas e isocinéticas, sendo que todos os mecanismos que o desencadeiam não estão ainda completamente elucidados, o que torna o tema atraente para 
a investigação científica. Com isso, seria interessante que outros estudos investiguem esses mecanismos e ainda que mais estudos sejam realizados para verificar o efeito de cada tipo de condição, unilateral e bilateral, durante o treinamento de força. Assim, a utilização de cada tipo de treinamento poderá ser fundamentada e utilizada como uma ferramenta de incremento das adaptações geradas pelo treinamento de força. De acordo com o que tem sido observado nos diferentes estudos que investigam o tema, os exercícios unilaterais por permitirem o levantamento de maiores cargas parecem incrementar em maior magnitude a força e possivelmente as adaptações neurais e morfológicas, podendo ser utilizado como uma estratégia na prescrição do treinamento de força.

\section{REFERÊNCIAS BIBLIOGRÁFICAS}

1. Pinto RS, Botton CE, Kuckartz BT, Lima CS, Moraes AC, Bottaro M. Avaliação do déficit bilateral em contrações isométricas dos extensores de joelhos. Rev Bras Cineantropom Desempenho Hum 2012;4(2):202-11.

2. Owings TM, Grabiner MD. Normally aging older adults demonstrate the bilateral deficit during ramp and hold contractions. J Gerontol: Biol Sci 1998;53(6):B425-B9.

3. Kuruganti U, Murphy T. Bilateral deficit expressions and myoelectric signal activity during submaximal and maximal isometric knee extensions in young, athletic males. Eur J Appl Physiol 2008;102:721-6.

4. Ohtsuki T. Decrease in human voluntary isometric arm strength induced by simultaneous bilateral exertion. Beh Brain Res 1983;7:165-78.

5. Oda S, Moritani T. Cross-correlation studies of movement-related cortical potentials during unilateral and bilateral muscle contractions in humans. Eur J Appl Physiol 1996;74:29-35.

6. Schantz PG, Moritani T, Karlson E, Johansson E, Lundh A. Maximal voluntary force of bilateral and unilateral leg extension. Acta Physiol Scand 1989;136:185-92.

7. Kuruganti U, Seaman K. The bilateral leg strength deficit is present in old, young and adolescent females during isokinetic knee extension and flexion. Eur J Appl Physiol 2006;97:322-6.

8. Howard JD, Enoka RM. Maximum bilateral contraction are modified by neurally mediated interlimb effects. J Appl Physiol 1991;70(1):306-16.

9. Vandervoort AA, Sale DG, Moroz J. Comparison of motor unit activation during unilateral and bilateral leg extension. J Appl Physiol 1984;56(1):46-51.

10. Henry FM, Smith LE. Simultaneous vs. separate bilateral muscular contractions in relation to neural overflow theory and neuromotor specificity. Res Q Exerc Sport 1961;32:42-7.

11. Owings TM, Grabiner MD. Fatigue effects on the bilateral deficit are speed dependent. Med Sci Sports Exerc 1998;30(8):1257-62.

12. Jakobi JM, Cafarelli E. Neuromuscular drive and force production are not altered during bilateral contractions. J Appl Physiol 1998;84:200-6.

13. Koh TJ, Grabiner MD, Clough, C A. Bilateral deficit is larger for step than for ramp isometric contractions. J Appl Physiol 1993;74(3):1200-5.

14. Häkkinen K, Kraemer WJ, Kallinen M, Linnamo V, Pastinen U-M, Newton RU. Bilateral and unilateral neuromuscular function and muscle cross-sectional area in middle-aged and elderly men and women. J Gerontol: Biol Sci 1996;51(1):B2I-B9.

15. Jakobi JM, Chilibeck PD. Bilateral and unilateral contractions: Possible differences in maximal voluntary force. Can J Appl Physiol 2001;26(t):12-33.

16. De Luca CJ. The use of electromyography in biomechanics. J Appl Biomech 1997;13:135-63. 
17. Kuruganti U, Chester V. Force production and neuromuscular function in bilateral movements among young females at low and high speeds. 25th Southern Biomedical Engineering Conference 2009. p.51-54.

18. Khodiguian N, Cornwell A, Lares E, Dicaprio P. A., HAWKINS, S. A. Expression of the bilateral deficit during reflexively evoked contractions. J Appl Physiol 2003;94:171-8.

19. Van Dieën JH, Ogita F, De Haan A. Reduced neural drive in bilateral exertions: a performance-limiting factor? Med Sci Sports Exerc 2003;35(1):111-8.

20. Matkowski B, Matin A, Lepers R. Comparison of maximal unilateral versus bilateral voluntary contraction force. Eur J Appl Physiol 2011;11(8):1571-8.

21. Kellis E. Quantification of quadriceps and hamstring antagonist activity. Sports Med 1998;25s(1):37-62.

22. Kuruganti U, Murphy P, Pardy T. Bilateral deficit phenomenon and the role of antagonist muscle activity during maximal isometric knee extensions in young, athletic men. Eur J Appl Physiol 2011;111:1533-9.

23. Kawakami Y, Sale DG, Macdougall JD, Moroz JS. Bilateral deficit in plantar flexion: relation to knee joint position, muscle activation, and reflex excitability. Eur J Appl Physiol 1998;77:212-6.

24. Kuruganti U, Parker PA, Tingley M, Sleivert GG. A comparison of measures of the bilateral limb deficit during short and long time isometric knee extensions. The Internet Journal of Bioengineering 2010;4(2). DOI: 10.5580/1090.

25. González-Izal M, Malanda A, Gorostiaga E, Izquierdo M. Electromyographic models to assess muscle fatigue. J Electromyogr Kinesiol 2012; 22(4):501-12 .

26. Lowery MM, Vaughan CL, Nolan PJ, O'Malley MJ. Spectral compression of the electromyographic signal due to decreasing muscle fiber conduction velocity. IEEE Trans Rehabil Eng 2000;8(3):353-61.

27. St Clair Gibson A, Lambert MI, Noakes TD. Neural control force output during maximal and submaximal exercise. Sports Med 2001;31(9):637-50.

28. Hernandez JP, Nelson-White NL, Franke WD, McLean SP. Bilateral index expressions and iEMG activity in older versus younger adults. J Gerontol A Biol Sci Med Sci 2003;58:M536-M41.

29. Secher NH, Rorsgaard S, Secher O. Contralateral influence on recruitment of curarized muscle fibers during maximal voluntary extension of the legs. Acta Physiol Scand 1978;103:456-462.

30. Kuruganti U, Parker P, Rickards J, Tingley M, Sexsmith J. Bilateral isokinetic training reduces the bilateral leg strength deficit for both old and young adults. Eur J Appl Physiol 2005;94:175-9.

31. Janzen CL, Chilibeck PD, Davison KS. The effect of unilateral and bilateral strength training on the bilateral deficit and lean tissue mass in post-menopausal women. Eur J Appl Physiol 2006;97:253-260.

32. Taniguchi Y. Lateral specificity in resistance training: the effect of bilateral and unilateral training. Eur J Appl Physiol 1997;75:144-50.

33. Häkkinen K, Kallinen M, Linnamo V, Pastinen U M, Newton RU, Kraemer WJ. Neuromuscular adaptations during bilateral versus unilateral strength training in middle-aged and elderly men and women. Acta Physiol Scand 1996;158:77-88.

34. McCurdy KW, Langford GA, Doscher MW, Wiley LP, Mallard KG. The effects of short-term unilateral and bilateral lower-body resistance training on measures of strength and power. J Strength Cond Res 2005;19(1):9-15.

35. Folland JP, Williams AG. The adaptations to strength training.morphological and neurological contributions to increased strength. Sports Med 2007;37(2):145-68.

36. Bird SP, Tarpenning KM, Marino FE. Designing resistance training programmes to enhance muscular fitness a review of the acute programme variables. Sports Med 2005;35(10):841-51.

Endereço para correspondência

Cíntia Ehlers Botton

LAPEX, Universidade Federal do Rio Grande do Sul

Rua Felizardo, 750, Jardim Botânico CEP: 90690-200 - Porto Alegre, RS. Brasil

E-mail: cintiabotton@yahoo.com.br 